\title{
The clinical characteristics of pregnant women with epilepsy in China
}

\author{
Chun-Yu Huang ${ }^{1}$, Yin-Mei Dai ${ }^{1}$, Li-Min Feng ${ }^{2}$, Wan-Li Gao ${ }^{2}$ \\ ${ }^{1}$ Department of Gynecology, Beijing Obstetrics and Gynecology Hospital, Capital Medical University, Beijing, China; ${ }^{2}$ Department of Obstetrics and \\ Gynecology, Beijing Tiantan Hospital, Capital Medical University, Beijing, China \\ Contributions: (I) Conception and design: CY Huang, YM Dai; (II) Administrative support: LM Feng; (III) Provision of study materials or patients: \\ WL Gao; (IV) Collection and assembly of data: LM Feng, WL Gao; (V) Data analysis and interpretation: LM Feng, WL Gao; (VI) Manuscript \\ writing: All authors; (VII) Final approval of manuscript: All authors. \\ Correspondence to: Yin-Mei Dai. Department of Gynecology, Beijing Obstetrics and Gynecology Hospital, Capital Medical University, No. 251, \\ Yaojiayuan Road, Chaoyang District, Beijing, China. Email: fcyydym@163.com; Li-Min Feng. Department of Obstetrics and Gynecology, Beijing \\ Tiantan Hospital, Capital Medical University, No. 119, Nansihuanxilu, Fengtai District, Beijing, China. Email: lucyfeng1966@163.com.
}

\begin{abstract}
Background: Epilepsy during pregnancy and puerperium is infrequent, and it can induce severe complications and poor prognosis. Pregnancy in women with epilepsy (WWE) is usually uneventful. Previous studies have mainly focused on the effects of different treatments on prognosis. However, few articles have addressed if different epilepsy types were associated with a higher incidence of seizure breakthrough/recurrence and pregnancy outcomes.

Methods: In the present study, based on a unique sample with a low incidence of epilepsy, we evaluated the main clinical characteristics of epilepsy patients.

Results: Mean age of pregnant WWE was 29.95 4.65 (range, 21-42) years. Pregnancies were at a mean gestational age of $33.80 \pm 9.14$ (range, 7-41) weeks, and 85.24\% (52/61) of WWE were in their third trimester. There was $9.84 \%(6 / 61)$ of pregnant WWE underwent abortion or induced labor in midpregnancy to ensure maternal safety. There was $75.41 \%$ (46/61) of pregnant WWE using antiepileptic drugs (AEDs), of which 52.46\% (32/61) were taking a single AED and 22.95\% (14/61) were using multiple AEDs. There was 47.54\% (29/61) of WWE experiencing seizures during their pregnancy. We found that the type of epilepsy did not affect seizures during pregnancy or the prognosis. However, more pregnant WWE with hypertensive disorder had seizures compared with pregnant WWE without hypertensive disorder.
\end{abstract}

Conclusions: The study highlighted a novel direction for effectively improving seizures during pregnancy and the prognosis of pregnancy-associated epilepsy.

Keywords: Pregnancy; epilepsy; pregnancy outcome

Submitted Sep 29, 2019. Accepted for publication Jul 31, 2020.

doi: 10.21037/apm-19-341

View this article at: http://dx.doi.org/10.21037/apm-19-341

\section{Introduction}

Approximately $6.1 \%$ of pregnant women have a history of epilepsy (1). Pregnancy in women with epilepsy (WWE) is usually uneventful (2), however, complications that impact maternal and fetal health can occur (3). Most studies in pregnant WWE have focused on antiepileptic drug (AED) therapy due to the risk of teratogenic effects on the developing fetus. Few studies have described the demographic and clinical characteristics of pregnant WWE and investigated the influence of the type of epilepsy and hypertensive disorder of pregnancy (HDP) on complications in pregnant WWE.

The objective of this study was to describe the demographic and clinical characteristics of pregnant WWE and evaluate maternal and fetal outcomes, which will provide 
Table 1 Clinical characteristics and pregnancy outcomes of pregnant WWE $(\mathrm{n}=61)$

\begin{tabular}{|c|c|}
\hline Variables & Value \\
\hline Age (years) & $29.95 \pm 4.65$ \\
\hline Gestational age (weeks) & $33.80 \pm 9.14$ \\
\hline Gravidity & $1.77 \pm 1.00$ \\
\hline Parity & $1.20 \pm 0.65$ \\
\hline \multicolumn{2}{|l|}{ AED use, $\%[n]$} \\
\hline Monotherapy & $52.46[32]$ \\
\hline Polytherapy & $22.95[14]$ \\
\hline Did not use AEDs & $24.59[15]$ \\
\hline Incidence of seizures & $47.54[29]$ \\
\hline \multicolumn{2}{|l|}{ Type of epilepsy, \% [n] } \\
\hline Genetic & $67.21[41]$ \\
\hline Non-genetic & $32.79[20]$ \\
\hline \multicolumn{2}{|l|}{ Gestational age, \% [n] } \\
\hline Early pregnancy & $8.20[5]$ \\
\hline Second trimester & $6.56[4]$ \\
\hline Third trimester & $85.24[52]$ \\
\hline \multicolumn{2}{|l|}{ Maternal complications, \% [n] } \\
\hline HDP & $14.75[9]$ \\
\hline Mortality & $1.64[1]$ \\
\hline \multicolumn{2}{|l|}{ Perinatal outcomes } \\
\hline Malformation, \% [n] & $1.64[1]$ \\
\hline Abortion and induced labor, \% [n] & $9.84[6]$ \\
\hline Prematurity, \% [n] & $13.11[8]$ \\
\hline Term delivery, $\%[\mathrm{n}]$ & $75.41[46]$ \\
\hline Intrauterine death, $\%[n]$ & $1.64[1]$ \\
\hline Mortality, \% [n] & $1.64[1]$ \\
\hline Mean newborn weight (g) & $3,123.37 \pm 610.2$ \\
\hline Average Apgar score of the newborns & $9.71 \pm 0.77$ \\
\hline \multicolumn{2}{|l|}{ Mode of delivery, \% [n] } \\
\hline Cesarean section & $80.33[49]$ \\
\hline Vaginal delivery & $19.67[12]$ \\
\hline
\end{tabular}

WWE, women with epilepsy; HDP, hypertensive disorder of pregnancy.

pre-pregnancy counseling and management in WWE, as well as prenatal and postpartum care of pregnant WWE.

We present the following article in accordance with the STROBE reporting checklist (available at http://dx.doi. org/10.21037/apm-19-341).

\section{Methods}

The study conformed to the provisions of the Declaration of Helsinki (as revised in 2013). This study was approved by Institutional Research Ethics Committee of Beijing Tiantan Hospital (No. KY 2019-029-02). Requirements for written informed consent were waived because all personal data were de-identified before the analyses.

Medical records of pregnant women with a history of epilepsy who attended the Beijing Tiantan Hospital, China, from January 2004 to December 2017 were retrospectively reviewed. Inclusion criteria were: (I) in-hospital delivery at Beijing Tiantan Hospital; and (II) a diagnosis of epilepsy according to the 2014 International League Against Epilepsy (ILAE) criteria (4).

Maternal and fetal demographic and clinical characteristics and pregnancy outcomes were recorded. In subgroup analyses, the impact of the etiology of epilepsy on maternal and fetal outcomes was explored by stratifying the study sample according to genetic $v s$. non-genetic basis of epilepsy. A genetic basis of epilepsy was assumed from family history or characteristic signs and symptoms. The impact of HDP (pre-eclampsia, eclampsia, gestational hypertension, and chronic hypertension) on maternal and fetal outcomes was explored by stratifying the study sample based on the presence or absence of HDP, diagnosed according to American College of Obstetricians and Gynecologists criteria (5).

Statistical analyses were performed using SPSS version 20. Between group differences for data with a normal distribution were evaluated with unpaired $t$-tests. Comparisons of categorical data were performed with the chi-squared test. $\mathrm{P}<0.05$ was considered as statistically significant.

\section{Results}

\section{Demographic and clinical characteristics WWE}

The medical records of 61 pregnant WWE were retrospectively reviewed. During study period, $0.25 \%$ $(61 / 23,721)$ of pregnant women attending the Beijing Tiantan Hospital had a history of epilepsy. Baseline demographic and clinical characteristics of the study samples were shown in Table 1. Mean age of pregnant WWE was $29.95 \pm 4.65$ (range, 21-42) years. Pregnancies were at a mean gestational age of $33.80 \pm 9.14$ (range, 7-41) weeks, and 85.24\% (52/61) of WWE were in their third trimester. There was $9.84 \%$ (6/61) of pregnant WWE underwent 
Table 2 Admission data and outcomes according to type of epilepsy

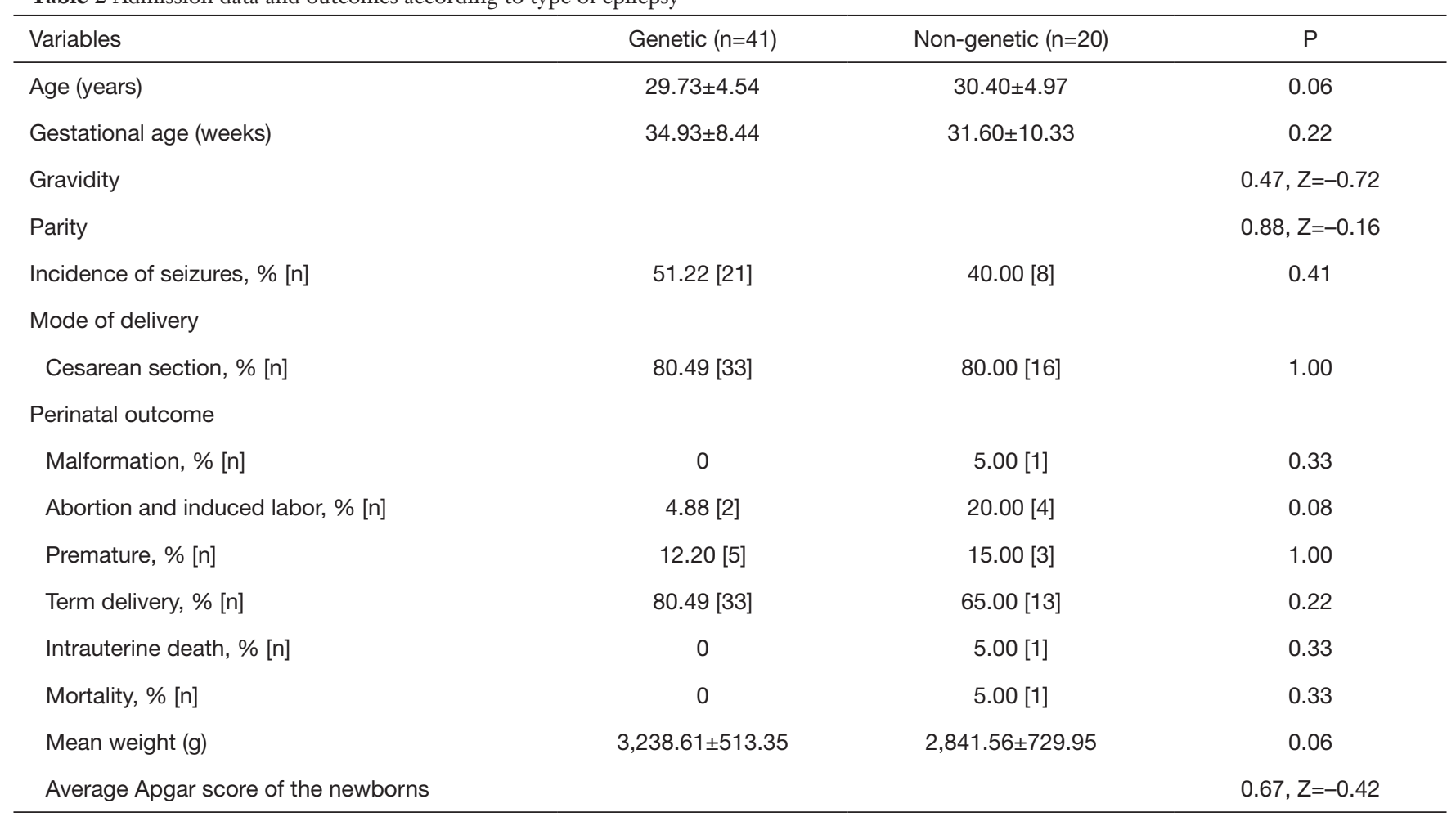

abortion or induced labor in mid-pregnancy to ensure maternal safety. There was $75.41 \%(46 / 61)$ of pregnant WWE using AEDs, of which 52.46\% (32/61) were taking a single AED and $22.95 \%$ (14/61) were using multiple AEDs. There was $47.54 \%$ (29/61) of WWE experiencing seizures during their pregnancy.

Epilepsy was genetic in $67.21 \%$ (41/61) of pregnant WWE. There were no significant differences in maternal variables, including age, median gestational age, gravidity, parity, or the incidence of seizures in pregnant women with a genetic or non-genetic basis for their epilepsy. Similarly, there were no significant differences in mode of delivery or perinatal outcomes (Table 2).

There was $14.75 \%$ (9/61) of pregnant WWE experienced HDP. The incidence of seizures was significantly higher in pregnant WWE with HDP compared with those without $(\mathrm{P}=0.01)$. There were no significant differences in maternal variables, including age, median gestational age, gravidity, or parity, or in mode of delivery or perinatal outcomes (Table 3).

\section{Discussion}

This study described the demographic and clinical characteristics of pregnant WWE. Mean age of pregnant WWE was less than 30 years. The majority of pregnant WWE used AED therapy during their pregnancy, and approximately $50 \%$ experienced seizures. Maternal and fetal outcomes were compared in pregnant WWE stratified by genetic or non-genetic basis of epilepsy and presence or absence of HDP. Type of epilepsy did not appear to affect maternal and fetal outcomes, but the incidence of seizures was significantly higher in pregnant WWE with HDP compared with those without.

Epilepsy is a chronic brain disorder characterized by the sudden onset of seizures caused by sudden abnormal neuronal firing. To our knowledge, the present study includes the largest sample of Chinese pregnant WWE reported to date. The study was conducted at Beijing Tiantan Hospital, a medical transport center for pregnant and postpartum patients with critical craniocerebral disease. The proportion of patients with epilepsy was expected to be higher than in other hospitals in Beijing. The incidence rate $(0.25 \%)$ is lower than that reported for the UK, where an estimated $0.6 \%$ of pregnancies were in $\mathrm{WWE}$, according to the 2011 report of the United Kingdom Confidential Enquiries into Maternal Deaths (6). 
Table 3 The impact of HDP on maternal and fetal outcomes

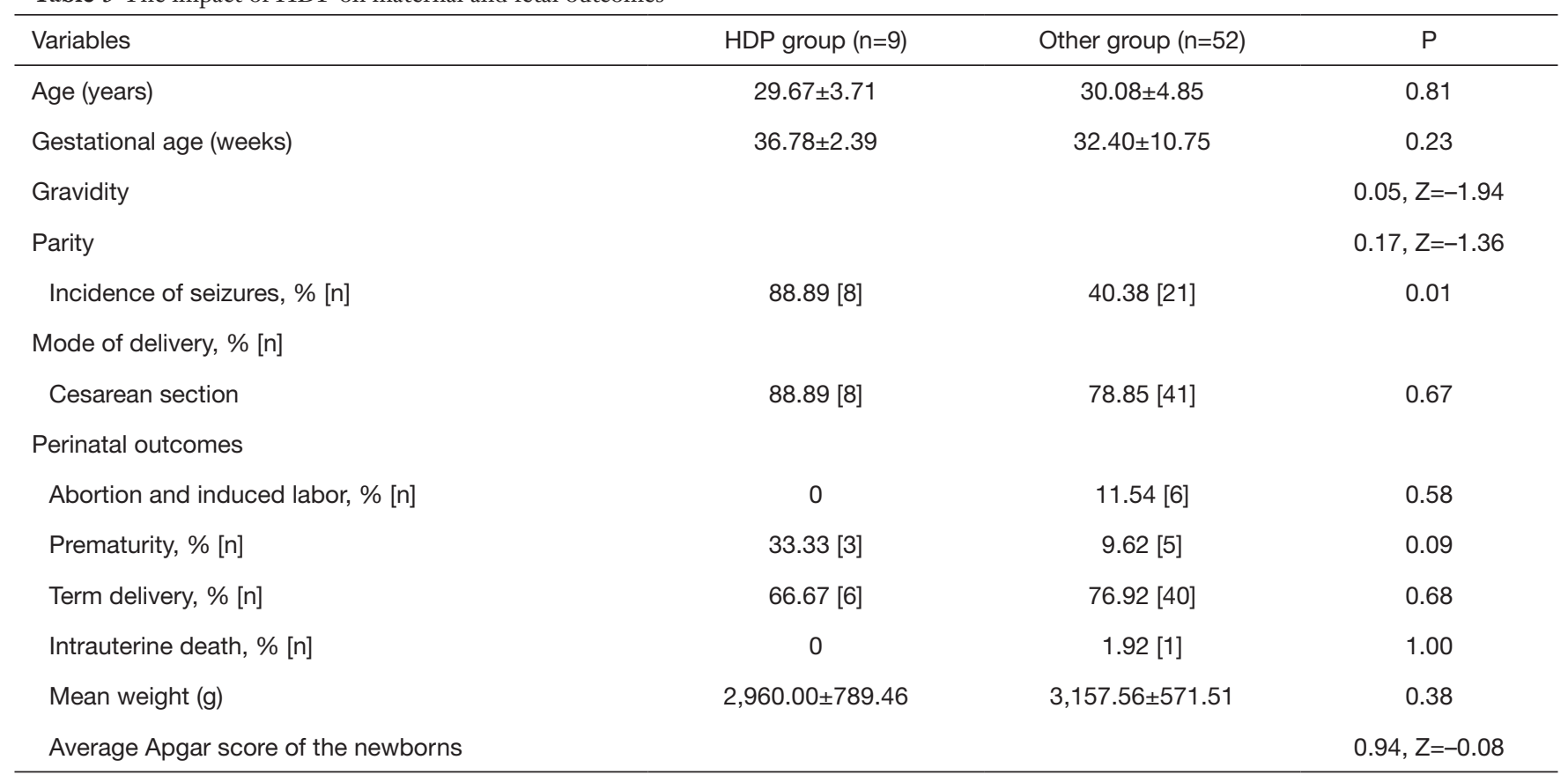

HDP, hypertensive disorder of pregnancy.

Pregnant WWE have an increased risk of adverse events compared with those without epilepsy, including preeclampsia, preterm labor, stillbirth, spontaneous abortion, vaginal bleeding, hypertension, postpartum hemorrhage, placental abruption, and an increased risk of cesarean delivery. Severe cases may even result in maternal death (7). In the present study, we reported one maternal death, for a case mortality rate of $1.64 \%$. A previous retrospective cohort study conducted using the 2007-2011 Nationwide Inpatient Sample in the US found that pregnant WWE had a 10 -fold increased risk of death compared with those without epilepsy (3).

The seizures that characterize epilepsy and the use of AEDs can result in poor maternal, fetal, and neonatal outcomes. Maternal seizures can not only cause maternal injury and obstetric sequelae like placental abruption, premature labor, and delivery, but also lead to hypoxia in the fetus and transient late decelerations, decreased beatto-beat variability, and compensatory tachycardia (8). AEDs can have teratogenic and long-term neurodevelopmental effects on the fetus. Taken together, these data emphasize the importance of appropriate preconception consultation, counseling, and planning of WWE and their clinicians to ensure epilepsy and seizures are safely managed during pregnancy.
Several factors increase the frequency of seizures during pregnancy. Metabolic changes during pregnancy alter the pharmacokinetics of AEDs, increasing volume of distribution, renal clearance, and hepatic metabolism (9). Physiological changes during pregnancy, such as sodium retention and edema, can lead to HDP. These alterations can also increase brain irritability so that minimal sensory stimuli, including fatigue, nervousness, and emotional changes, can trigger seizures. In our study, $14.75 \%$ of pregnant WWE experienced HDP, and the incidence of seizures in pregnant WWE with hypertensive disorder was significantly higher than those without. The incidence of hypertension in pregnant women without seizures has been reported to be about $13.2 \%$ in a previous study (10).

These findings indicated that clinicians should make every effort to monitor and treat HDP in WWE. Neurologists and obstetricians should work together, taking a multidisciplinary approach to the management of pregnant WWE, to optimize pregnancy outcomes.

As this is a retrospective study, we could not confirm the causal relationship of hypertensive disorder and the incidence of seizures. A previous study has showed that neuropathological complications were associated with hypertensive disorders of pregnancy (11). Unfortunately, there are no reliable predictors of seizures during 
pregnancy (2). Therefore, seizures should be controlled before pregnancy, and individualized therapeutic levels of AEDs should be established with monotherapy used where possible. WWE who are seizure-free in the 9 months prior to pregnancy have an $84-92 \%$ chance of remaining seizurefree during pregnancy on their current regimen (12). In our study, $52.46 \%$ of women remained seizure-free throughout pregnancy. These findings were consistent with previous studies that reported $58.3-66.6 \%$ of WWE participating in EURAP, the registry of international AEDs and pregnancy, were seizure-free throughout pregnancy $(13,14)$. The risk of seizure during pregnancy corresponds to seizure type; WWE with complex partial seizures are more likely to experience seizures during pregnancy (15).

There were several limitations in this study. First, the sample size was not large enough to allow broad generalization of the results across different healthcare settings and regions. Second, we might have underestimated the proportion of pregnant WWE among the pregnant women attending the Beijing Tiantan Hospital, as cases may have been missed during screening. Third, the current study did not investigate the long-term outcomes of newborns. Fourth, brain images and EEGs were not performed in all patients to differentiate genetic and non-genetic epilepsies. Fifty, we did not set an internal control group to show the incidence of hypertension in pregnant women without seizures.

In conclusion, this study described the demographic and clinical characteristics of pregnant WWE, as well as the maternal and fetal outcomes. We found there were no significant differences in maternal or fetal variables, including age, median gestational age, gravidity, parity, incidence of seizures, mode of delivery or perinatal outcomes between pregnant women with a genetic and non-genetic basis for their epilepsy. The incidence of seizures was significantly higher in pregnant WWE with hypertensive disorder than those without. These data highlight the need that neurologists and obstetricians should work together to adopt a multidisciplinary approach to manage pregnant WWE in order to optimize pregnancy outcomes.

\section{Acknowledgments}

The wordings of the main text and/or figures/tables have been checked by a native English-speaking expert and we appreciate the assistant of the following web https://fanyi. baidu.com/translate.
Funding: None.

\section{Footnote}

Reporting Checklist: The authors have completed the STROBE reporting checklist. Available at http://dx.doi. org/10.21037/apm-19-341

Data Sharing Statement: Available at http://dx.doi. org/10.21037/apm-19-341

Conflicts of Interest: All authors have completed the ICMJE uniform disclosure form (available at http://dx.doi. org/10.21037/apm-19-341). The authors have no conflicts of interest to declare.

Ethical Statement: The authors are accountable for all aspects of the work in ensuring that questions related to the accuracy or integrity of any part of the work are appropriately investigated and resolved. The study conformed to the provisions of the Declaration of Helsinki (as revised in 2013). This study was approved Institutional Research Ethics Committee of Beijing Tiantan Hospital (No. KY 2019-029-02). Requirements for written informed consent were waived because all personal data were deidentified before the analyses.

Open Access Statement: This is an Open Access article distributed in accordance with the Creative Commons Attribution-NonCommercial-NoDerivs 4.0 International License (CC BY-NC-ND 4.0), which permits the noncommercial replication and distribution of the article with the strict proviso that no changes or edits are made and the original work is properly cited (including links to both the formal publication through the relevant DOI and the license). See: https://creativecommons.org/licenses/by-nc-nd/4.0/.

\section{References}

1. Fairgrieve SD, Jackson M, Jonas P, et al. Population based, prospective study of the care of women with epilepsy in pregnancy. BMJ 2000;321:674-5.

2. Zahn CA, Morrell MJ, Collins SD, et al. Management issues for women with epilepsy: a review of the literature. Neurology 1998;51:949-56.

3. MacDonald SC, Bateman BT, McElrath TF, et al. Mortality and morbidity during delivery hospitalization among pregnant women with epilepsy in the United 
States. JAMA Neurol 2015;72:981-8.

4. Fisher RS, Acevedo C, Arzimanoglou A, et al. ILAE official report: a practical clinical definition of epilepsy. Epilepsia 2014;55:475-82.

5. ACOG Committee on Practice Bulletins--Obstetrics. ACOG practice bulletin. Diagnosis and management of preeclampsia and eclampsia. Number 33, January 2002. Obstet Gynecol 2002;99:159-67.

6. Edey S, Moran N, Nashef L. SUDEP and epilepsy-related mortality in pregnancy. Epilepsia 2014;55:e72-4.

7. Viale L, Allotey J, Cheong-See F, et al. Epilepsy in pregnancy and reproductive outcomes: a systematic review and meta-analysis. Lancet 2015;386:1845-52.

8. Sarma AK, Khandker N, Kurczewski L, et al. Medical management of epileptic seizures: challenges and solutions. Neuropsychiatr Dis Treat 2016;12:467-85.

9. Reisinger TL, Newman M, Loring DW, et al. Antiepileptic drug clearance and seizure frequency during pregnancy in women with epilepsy. Epilepsy Behav 2013;29:13-8.

10. Adekomi AD, Moodley J, Naicker T. Neuropathological complications associated with hypertensive disorders of pregnancy. Hypertens Pregnancy 2019;38:171-5.

11. Chen D, Lao M, Cai X, et al. Hypertensive disorders of

Cite this article as: Huang CY, Dai YM, Feng LM, Gao WL. The clinical characteristics of pregnant women with epilepsy in China. Ann Palliat Med 2020;9(5):2442-2447. doi: 10.21037/apm-19341 pregnancy associated with adverse pregnant outcomes in patients with systemic lupus erythematosus: a multicenter retrospective study. Clin Rheumatol 2019;38:3501-9.

12. Harden CL, Meador KJ, Pennell PB, et al. Practice parameter update: management issues for women with epilepsy--focus on pregnancy (an evidence-based review): teratogenesis and perinatal outcomes: report of the Quality Standards Subcommittee and Therapeutics and Technology Assessment Subcommittee of the American Academy of Neurology and American Epilepsy Society. Neurology 2009;73:133-41.

13. Battino D, Tomson T, Bonizzoni E, et al. Seizure control and treatment changes in pregnancy: observations from the EURAP epilepsy pregnancy registry. Epilepsia 2013;54:1621-7.

14. EURAP Study Group. Seizure control and treatment in pregnancy: observations from the EURAP epilepsy pregnancy registry. Neurology 2006;66:354-60.

15. Fröscher W, Herrmann R, Niesen M, et al. The course of pregnancy and teratogenicity of antiepileptic agents in 66 patients with epilepsy. Schweiz Arch Neurol Psychiatr (1985) 1991;142:389-407. 